\title{
In vitro generation of mouse polarised embryo-like structures from embryonic and trophoblast stem cells
}

Sarah Ellys Harrison ${ }^{1,3}$, Berna Sozen ${ }^{1,2,3}$, and Magdalena Zernicka-Goetz ${ }^{1 *}$

${ }^{1}$ Mammalian Embryo and Stem Cell Group, University of Cambridge, Department of Physiology,

Development and Neuroscience; Downing Street, Cambridge, CB2 3DY, UK

${ }^{2}$ Department of Histology and Embryology, Faculty of Medicine, Akdeniz University, Antalya, 07070, Turkey

${ }^{3}$ Equal contribution

*corresponding author

\section{Corresponding author email: mz205@cam.ac.uk}

Keywords: Embryonic stem cells, Trophoblast stem cells, embryogenesis, mouse embryo, proamniotic cavity, mesoderm. Primordial germ cells

Key references:

1) Harrison, S. E., Sozen, B., Christodoulou, N., Kyprianou, C. \& Zernicka-Goetz, M. (2017) 'Assembly of embryonic and extra-embryonic stem cells to mimic embryogenesis in vitro', Science. doi: 10.1126/science.aal1810.

2) Bedzhov, I. and Zernicka-Goetz, M. (2014) 'Self-Organizing Properties of Mouse Pluripotent Cells Initiate Morphogenesis upon Implantation.', Cell. Elsevier Inc., 156(5), pp. 1032-44. doi: 10.1016/j.cell.2014.01.023.

EDITORIAL SUMMARY:

Directing assembly of extra-embryonic trophoblast stem cells with embryonic stem cells to recapitulate post-implantation mouse embryogenesis.

\section{SUMMARY}

Mammalian embryogenesis requires the coordination of embryonic and extra-embryonic tissues to enable implantation into the uterus and post-implantation development to establish the body plan. Mouse embryonic stem cells (ESCS) are a useful tool for studying pluripotent embryonic tissue in vitro. However, they cannot undertake correct embryogenesis alone. Many attempts to model the early embryo in vitro involve the aggregation of ESCs into spheroids of variable size and cell number that undertake germ-layer specification but fail to recapitulate the characteristic architecture and arrangement of tissues of the early embryo. Here, we describe a protocol to generate the first embryo- 
like structures by directing the assembly of mouse ESCs and extra-embryonic trophoblast stem cells (TSCS) in a 3D extracellular matrix (ECM) into structures we call 'polarized embryo-like structures'. By establishing the medium and culture conditions needed to support the growth of both stem cell types simultaneously, embryonic architecture is generated within $4 \mathrm{~d}$ of co-culture. This protocol can be performed by those proficient in standard ESC culture techniques and can be used in developmental studies to investigate the interactions between embryonic and extra-embryonic tissues during mammalian development.

\section{INTRODUCTION}

At implantation, the mouse embryo, known as a blastocyst, is made up of three distinct tissues, which are arranged in a specific configuration to develop into a structure known as the egg cylinder. One of these tissues is the pluripotent embryonic epiblast, which gives rise to the fetus and forms a cupshaped epithelium at the distal side of the conceptus. Abutting the epiblast is one of two extraembryonic tissues, the extra-embryonic ectoderm (ExE), which is derived from the trophectoderm and positioned at the proximal side of the embryo, giving rise to the fetal portion of the placenta. The third tissue, the visceral endoderm (VE), gives rise to the yolk sac of the embryo. This forms a monolayer of cells that envelops the other two tissue compartments. These extraembryonic tissues not only nourish the embryo as it grows, they also provide essential signals to pattern the epiblast, ensuring that the body plan is established correctly. In this protocol, we describe a system to model the early development of the mouse embryo ex vivo that utilizes cells derived from both embryonic and extra-embryonic tissues to mimic in vivo embryogenesis.

\section{Modelling embryogenesis with ESCs alone}

Mouse embryonic stem cells (ESCS) can be derived from the pre-implantation blastocyst and are transcriptionally equivalent to the epiblast of the mouse embryo just before implantation, at embryonic day $4.5(E 4.5)^{1}$. These cells are a useful tool to investigate the early development of the pluripotent embryonic tissue in the first few days of embryogenesis in vitro. However, when allowed to differentiate in $2 \mathrm{D}$ adherent culture, these cells cannot undertake embryogenesis without the support of the in vivo environment. Instead, they differentiate into neural tissue ${ }^{2}$ or can be coaxed into generating other lineages by the administration of growth factors to bias cell fate ${ }^{3,4}$. When cultured in 3D with extracellular matrix (ECM), mammalian ESCs and their reprogrammed counterparts, induced pluripotent stem cells have been shown to develop with time into organ-like structures including fully organised retina and brain-like tissue $e^{5-9}$. The generation of these structures, termed 'organoids', occurs through self-assembly/self-organisation of cells from an initially 
homogenous pluripotent population. Whilst some organoid structures exhibit endogenous axial patterning ${ }^{6,10}$, ESCs in culture fail to reproducibly establish body axes without exogenous signals and do not generate a structure resembling whole-embryonic architecture. Initial attempts to model early post-implantation mouse embryogenesis focussed on the use of ESCs alone to generate 3D aggregates of hundreds of cells. This technique leads to the formation of 'embryoid bodies' that can spontaneously induce domains of polarised gene expression ${ }^{11}$. Some of these structures have been termed 'gastruloids' as they sometimes initiate the regionalised expression of germ layer markers associated with the onset of gastrulation during embryogenesis ${ }^{12,13}$. However, these structures do not develop as embryos and, consequently, do not recapitulate the specific architecture of the embryo at post-implantation stages, nor do they represent the extra-embryonic lineages present in the mammalian embryo.

When embedded in ECM and when starting from individual cells, ESCs more faithfully mimic embryonic architecture. They develop to make a 'rosette-like' structure similar to the embryonic epiblast at the time of implantation ${ }^{14}$. Just as in the embryo, $\beta 1$-integrin signalling mediated by laminin in the ECM leads to cell polarisation, which in turn underpins the formation of the ESC 'rosette'. At the centre of the rosette, a lumen opens analogous to the opening of the pro-amniotic cavity during embryogenesis ${ }^{14}$.

\section{Development of the protocol}

Given that the embryo at early stages comprises extra-embryonic tissues in addition to the embryonic epiblast, the extra-embryonic ectoderm (ExE) and the visceral endoderm (VE) and these have been demonstrated to be important for establishing the body plan ${ }^{15}$, we introduced trophoblast stem cells (TSCS) derived from the ExE of the embryo ${ }^{16}$ into co-culture with ESCs embedded in ECM ${ }^{17}$. We also developed a medium to support the culture of ESCS and TSCs simultaneously, and used ECM as a substitute for the basement membrane secreted by the VE, which we showed previously to be critical for epiblast polarisation and lumenogenesis in vivo ${ }^{14}$.

We demonstrated that ESCs and TSCs in this system could self-assemble into structures that mimicked embryo architecture within 4 days of culture ${ }^{17}$. These structures underwent similar morphogenesis to give rise to a cavity akin to the pro-amniotic cavity in the mouse egg cylinder, which is shared between the embryonic and extra-embryonic tissues. Despite the fact that only $22 \%$ of all structures formed comprised both ESCs and TSCs; when these 2 types of stem cell met, more than $90 \%$ of the structures developed a characteristic embryo-like morphology demonstrating the robustness of this model system ${ }^{17}$. Here we provide a step-by-step protocol for generating these 
'polarised embryo-like structures', also called 'ETS-embryos' (ESCS and TSCs in culture), using custom developed media, ECM and optical plastic for high-resolution imaging of structures. This method demonstrates a new tool that can be used to investigate early mouse embryogenesis using a tractable stem cell model.

\section{Potential applications of polarised embryo-like structures and comparison to other methods}

We showed that our polarised embryo-like structures (ETS-embryos) recapitulate proamniotic cavity formation in the mouse embryo and therefore can be used to study how this cavity develops (Fig. 1g-h). They also recapitulate cell fate decisions and axial patterning events associated with early post-implantation development ${ }^{17}$. We showed that they can develop an area of regionalised mesoderm at the boundary between ESC and TSC compartments, which is analogous to the domain which marks the onset of gastrulation at the proximo-posterior side of the epiblast ${ }^{18}$. Opposite this region of mesoderm, cells acquire a more anterior-like fate, upregulating genes normally expressed in the anterior epiblast of the E6.5 embryo, such as Pou3f1, Oct4, S/c7a3 and Utf1 ${ }^{17,19,20}$. This indicates changes in the transcriptional program that reflects a gradient of cell fates across the embryonic compartment. Further development of these polarised embryo-like structures leads to specification of a small number of Primordial Germ Cell (PGC)-like cells. Amazingly, these PGC-like cells become specified in the correct spatial location, at the boundary between ESC and TSC compartments, within the T/Brachyury-positive domain ${ }^{17}$. We showed that this PGC-like cell specification occurs via BMP signalling, as it does in the embryo, and both induction of PGC and mesoderm markers in are preceded by the localised induction of $\mathrm{Wnt}^{21,22}$. Thus, polarised embryo-like structures mimic the spatial and temporal events of early mouse embryogenesis ${ }^{17}$.

In addition to being a useful tool to study cavitation in the embryo, because polarised embryolike structures can break symmetry and specify mesoderm in a regionalised manner, these structures can be used to study how mesoderm becomes specified. In comparison to embryoid bodies and the gastruloids derived from them, ETS-embryos stand out, in that both whole embryo architecture and germ layer specification occur as a result of the activity of endogenous signals provided by the TSCderived extra-embryonic compartment. This is in contrast to coaxing ESCs in 3D aggregates to differentiate by supplementing the culture medium with growth factors such as recombinant Wnt or $B M P^{11-13}$. In this respect, ETS embryos are useful for the study of how embryonic-and extra-embryonic tissues interact in the embryo to specify embryo architecture and the future body axes. Furthermore, the identity and role of endogenous signals in the these embryo-like structures may be deduced by adding inhibitors of known morphogens to the culture system, and monitoring the effects on mesoderm specification. 
Embryoid bodies and gastruloids which are derived from them comprise only one cell type. As such, they may be useful in the study of how ESCs differentiate in response to the delivery of an exogenous signal. Moreover, although polarised embryo-like structures lack a VE layer, which is critical for natural embryo development, the fact that they are more simple than the real embryo but still comprise embryonic and extra-embryonic tissues aids investigation of the interaction between the epiblast and the ExE. This is because the absence of the VE means it cannot confound results by producing its own signals which affect embryogenesis.

In addition to being a valuable tool to investigate the patterning of the body axes, ETS embryos also have several other potential uses in developmental studies. This in vitro model of the mouse embryo can complement work in vivo as the amenability of ESCs and TSCs to genetic manipulation means that they can be used to generate embryo-like structures that are knockout for a particular gene of interest. This may be an efficient way to reveal novel functions of genes in early embryogenesis, and to perform molecular studies, without producing knockout animals which is both expensive and laborious. This approach may also aid in the identification and study of 'hidden' developmental phenotypes that might be masked in the mouse as maternally produced protein can compensate when the zygotic genome is compromised. Furthermore, the tissue-specific role of a gene can be easily probed in this system by knocking it out in either the embryonic or extra-embryonic cell line only. Whilst tissue-specific knockouts of mice may require the generation of a construct including a tissue specific promoter, this can be avoided using this embryo-like model as constitutive promoter systems can be introduced into each cell line independently.

\section{Current limitations of the protocol and potential future directions}

By mixing ESCs and TSCs in suspension and allowing 3D structures to grow, the ETS embryo culture is heterogeneous. On average, $22 \%$ of all structures in a single well are comprised of both stem cell types, whereas the majority of other structures comprise only ESCs and $\mathrm{TSCs}^{17}$. Despite this, by plating the recommended cell numbers, a yield of $>100$ polarised embryo-like structures can be achieved from a single experiment in a single 8-well $\mu$-plate (Ibidi). This is more than one can analyse per day. We have not tried to optimise this method to generate embryo-like structures that would develop beyond early post-implantation stages. This may require culturing conditions allowing more efficient exchange of gas and nutrients to permit the growth of larger, more complex structures akin to embryos at later developmental stages. As polarised embryo-like structures are structurally robust enough to survive manual removal from the Matrigel after 4 days in culture, they could be maintained 
in suspension using a rotary orbital shaker, or rotation culture using a spinning bioreactor ${ }^{23}$. Alternatively, a perfusion bioreactor could support structures remaining embedded in ECM.

In addition, the protocol in its current form yields embryo-like structures without a layer to represent the VE, which is the precursor to the yolk-sac. One improvement would be to establish a protocol to include extra-embryonic stem cells derived from the primitive endoderm ('XEN' cells) into co-culture. To do this, the medium composition described here may need to be altered to support the growth and proliferation of all three cell types. Furthermore, it has been shown that XEN cells in conventional culture are more equivalent to the primitive endoderm of the pre-implantation mouse embryo ${ }^{24,25}$ but cells which are equivalent to the post-implantation VE lineage might be required. Several studies have shown that XEN cells can be coaxed to become more VE-like when treated with $B M P{ }^{26,27}$, and so it may be that XEN cells would require 'priming' towards a VE-like state in this way before they can be used to generate VE tissue in our embryo-like structures. The use of Matrigel in the current protocol partially compensates for the VE, as this tissue is known to secrete the basement membrane essential for epiblast polarisation and lumenogenesis ${ }^{14}$. If VE-like cells were to be incorporated into our embryo-like structures, it is likely that these cells will secrete ECM components, thus providing the culture with exogenous sources of ECM.

\section{Experimental design}

Medium optimisation. Our approach is based on that developed by Bedzhov \& ZernickaGoetz $^{14}$ in which epiblast architecture is mimicked using ESCs in ECM by introducing TSCs into the system. To develop a medium that would support both types of stem cells in these conditions, we began by mixing 25\% Neurobasal A, 25\% DMEM/F-12 (Gibco Life-Technologies) and 50\% MegaCell RPMI medium (Sigma) (vol/vol). We found that medium based on one of these components alone was not sufficient to support the growth of both cell types simultaneously in culture, and that the basal composition that would support ESC and TSC growth at the same time required RPMI medium, which is usually used for conventional TSC culture, to be mixed with Neurobasal-A and DMEM-F/12 media, which is used to make N2B27 for conventional ESC culture. If one or several of these basal components were missing, one or both cell types failed to grow and/or began to differentiate.

The medium we developed also included extra-nutrients to promote cell growth and survival in culture, including L-glutamine and sodium pyruvate (Gibco Life-Technologies). We supplemented this medium with FGF4 and heparin, to promote TSC self-renewal in vitro, but excluded the inhibitors PD0325901, CHIR99021, and Leukaemia Inhibitory Factor (LIF), which maintain ground state pluripotency in $\mathrm{ESCs}^{28}$. This was because we wanted ESCs to be able to differentiate in co-culture. This medium also included $10 \%$ (vol/vol) FBS. Serum contains undefined factors which promote cell 
proliferation and differentiation, and is required for mouse blastocysts to develop into egg cylinders in vitro ${ }^{29,30}$. We hypothesised that serum would provide essential signalling components to ESCs and TSCs in co-culture that might promote self-assembly of embryo-like structures, but we kept serum content low to minimise the possibility of precocious differentiation. If higher concentrations of serum were added to the culture, such as $20 \%$ or $30 \%$, ETS-embryo morphology was compromised and the structures failed to develop a cavity at their centre.

By keeping the serum concentration low in the medium, we found that this medium composition supported the proliferation and formation of rosette-like structures of both ESCs and TSCs in Matrigel, which later self-assembled into 3D structures that resembled the egg cylinder (Fig. 1). These structures more faithfully mimicked the structure of the embryo than embryoid bodies and had similar cell number and tissue volume to the mouse egg cylinder ${ }^{17}$.

Cells. The use of different cell lines does not affect the efficiency of ETS embryo generation. We have routinely used transgenic reporter ESCs in the co-culture system to report different aspects of ETS-embryo development including mesoderm specification ${ }^{31}$, PGC-like cell specification ${ }^{32}$ or to demarcate the ESC compartment by possession of fluorescent membranes ${ }^{33}$ (STEP 1). Cells can also be derived de novo from a transgenic mouse line-of-interest and used in this protocol. Additional information on the derivation and culture techniques of stem cells from the mouse embryo can be found in: 'Manipulating the Mouse Embryo: a Laboratory Manual'34. Notably, ESCs and TSCs can still form embryo-like structures even when derived from different mouse strains.

ECM support. We recommend the use of Growth Factor Reduced Matrigel Matrix, (STEP 13) however every batch of matrix must be individually tested in the system to ensure reproducibility. Matrigel lots and batches can be tested before ETS-embryo generation by embedding ESCs and TSCS separately and culturing for $72 \mathrm{~h}$ to ensure the cells grow as expected (STEPS 14-15). For ESCs embedded in Matrigel, a single lumen should develop at the centre of the structure within $48 \mathrm{~h}^{14}$ and the cells should express the epiblast markers Sox2 or Otx2. TSCs embedded in Matrigel should continue to express ExE markers such as Eomes or Tfap2C as described below (Fig.2).

Imaging. ETS-embryos can be fixed, stained and imaged in situ at any time-point after plating, (STEPS 16-22) but mesodermal lineages appear only after 4-5 days in culture (Fig.2) (see ANTICIPATED RESULTS). If live-imaging is required, we recommend plating all structures in a single plane using the '3D on top' method (see BOX 1). The '3D on top' method can be used with various dish sizes, depending on the requirements of the experiment. Plating volumes for example dishes (Mattek Glass Bottomed culture dishes and Ibidi angiogenesis $\mu$-plates) are included in Supplementary Table 1. 


\section{MATERIALS}

\section{REAGENTS}

- Mitotically inactivated mouse embryonic fibroblasts (MEFs) ready to use (R\&D systems, PSC001) or prepared from mouse embryos as described in Box 1.

- Mouse trophoblast stem cells (derived as described in Box 2).

- Mouse ESCs derived from the mouse embryo at blastocyst stages (as described in Box 3) or obtained commercially from suppliers such as The Jackson Laboratory.

- DMEM/F-12 (Gibco- Life Technologies, Cat No 21331-020)

- DMEM (+ L-glutamine) (Gibco- Life Technologies Cat. No. 41966052)

- Neurobasal A liquid (Gibco- Life Technologies Cat. No. 10888-022)

- MegaCell RPMI-1640 medium (Sigma Aldrich Cat. No. M3817)

- $\quad$ N2 supplement (Made as described in RSU)

CRITICAL: Home-made N2 supplement yields better resuts than commercial N2 (Thermo Fisher Scientific Cat. No. 17502048). The use of some brands of commercial N2 can result in ETS-embryos with smaller or no cavities. Before using commercial N2 in this protocol, batch test the commercial product by using it to make N2B27 medium (described in 'Reagent setup') and use this to culture ESCs embedded in Matrigel. ESC cysts should make lumens within $48 \mathrm{~h}$.

- Commercial ETS-embryo Medium (Cell Guidance Systems Cat. No. M13-25)

- Human Apo-tansferrin, cell culture tested (Sigma Aldrich Cat. No. T1147-100MG)

- Bovine Albumin Fraction V (7.5\% solution, (wt/vol)) (Gibco- Life Technologies Cat. No. 15260037)

- Chemically defined Human insulin solution (Sigma Cat. No. 19278-5ML)

- Putrescine dihydrochloride powder, cell culture tested (Sigma Aldrich Cat. No. P5780-5G)

- Sodium selenite powder, cell culture tested (Sigma Aldrich Cat. No. S5261-10G)

- Progesterone powder, cell culture tested (Sigma Aldrich Cat. No. P8783-1G)

- B27 supplement minus AO (Gibco- Life Technologies Cat. No. 10889-038)

- Embryonic stem cell grade foetal bovine serum (FBS) (Gibco- Life Technologies Cat. No. 10439024)

CRITICAL: FBS must be tested in ESC culture before use in the ETS embryo system. To do this, make up FC2iLIF medium (described in 'Reagent setup') and culture ESCs in adherent culture on gelatin in this medium for two weeks. Assess ESC growth and proliferation during this time, colony morphology, and expression of pluripotency markers. If the FBS is of appropriate quality, then ESCs should become confluent within $48 \mathrm{~h}$ of culture, and colonies should be rounded and raised, with tightly packed cells. Expression of pluripotency markers can be 
tested by immunofluorescence staining to detect expression of Oct4, Nanog, and Sox2 transcription factors, which should be localised to the nucleus. To check for differentiation, cells can also be immunostained for T/Brachyury protein, which should not be expressed in naïve pluripotent ESCs. The gold standard test for naïve pluripotent ESCS is to perform a chimera assay in which ESCs are introduced into the pre-implantation embryo and their contribution is checked. This is recommended for cell-lines which have been derived but not previously validated in this way.

- L-glutamine (Gibco- Life Technologies Cat. No. 25030-024) or Glutamax (Gibco- Life Technologies Cat. No. 35050-038)

- 2-Mercaptoethanol (2 ME) solution (50 mM) (Gibco- Life Technologies Cat. No. 31350-010) CAUTION: 2-Mercaptoethanol is toxic. Use personal protective equipment and discard waste appropriately.

- MEM Non-Essential Amino Acid Solution (NEAA) (Gibco- Life Technologies Cat. No. 11140035)

- Sodium pyruvate $100 \mathrm{mM}$ solution (Gibco-Life Technologies Cat. No. 11360039)

- Penicillin-streptomycin solution (Gibco-Life Technologies Cat. No. 15140122)

- PD0325901 (Stemgent, 04-0006)

- CHIR99021 (Stemgent, Cat. No. 04-0004)

- Recombinant mouse Leukaemia Inhibitory Factor (LIF) (Stemfactor, Cat. No. 03-0011)

- Recombinant Human Fibroblast Growth Factor 4 (FGF-4) (Peprotech Cat. No. 100-31)

- Heparin (Sigma Cat. No. H3149)

- Sterile Phosphate Buffered Saline (PBS) (Gibco-Life Technologies Cat. No. 10010056)

- Trypsin-EDTA (Gibco-Life Technologies Cat. No. 25300054)

- Growth Factor Reduced Matrigel matrix (BD, Corning Cat. No. 356234)

- Autoclaved water

- Ethanol solution (Sigma Aldrich Cat. No. 02860-4x25ml)

- Gelatin from cold water fish skin (Sigma Aldrich Cat. No. G7765)

- Bovine serum albumin (BSA) (Sigma Aldrich Cat. No. A3311-50G)

- Triton-X-100 (Sigma Aldrich Cat. No. T9284-100ML)

- $16 \%$ Paraformaldehyde (Electron Microscopy Sciences, Cat. No. 15710)

CAUTION: Paraformaldehyde is toxic. Use personal protective equipment and discard waste appropriately.

- Antibodies specific to Tfap2C (1:200; Santa Cruz, Cat. No. sc-8977), Brachyury/T (1:400; R\&D systems, Cat. No.AF2085), anti-GFP (1:1000; Nacalai Biochemicals, cat. No. 04404-84) Eomes 
(1:400; Abcam, Cat. No. ab183991), Sox2 (1:200; Santa Crux, Cat. No. sc-365823), Otx2 (1:200; R\&D systems, Cat. No. AF1979).

- Fluorophore conjugated secondary antibodies (1:500): donkey anti-goat Alexa Fluor 488 (Life Technologies, Thermofisher Scientific A11055), donkey anti-goat Alexa Fluor 568 (Life Technologies, Thermofisher Scientific A21447), donkey anti-rat Alexa Fluor 488 (Life Technologies, Thermofisher Scientific A21208), donkey anti-mouse Alexa Fluor 568 (Life Technologies, Thermofisher Scientific A10037), donkey anti-rabbit Alexa Fluor 647 (Life Technologies, Thermofisher Scientific A31573).

\section{EQUIPMENT}

- ibiTreat $\mu$-plates, 8-well (Ibidi, Cat. No. 80826)

- Angiogenesis $\mu$-plate (Ibidi, Cat. No. IB-81506)

- Glass-bottomed -culture dish (MatTek, Cat. No. P35G-1.5-14-C)

- Nunclon Tissue culture plates, six well, 12 well (Thermofisher Scientific Cat No. 140675 \& 150628)

- Sterile Syringe Filters CA $0.2 \mu \mathrm{m}, 33 \mathrm{~mm}$ (Gilson cat. No. ALG422A)

- Nikon inverted microscope for tissue culture

- Set of laboratory pipettes for tissue culture work

- $\quad \mathrm{P} 1000, \mathrm{P} 200, \mathrm{P} 20, \mathrm{P} 10, \mathrm{P} 2$ filter pipette tips

- $\mathrm{CO} 2$ incubator (New Brunswick, Galaxy $170 \mathrm{R}$ ) set at $5 \% \mathrm{CO} 2$ and $37^{\circ} \mathrm{C}$.

- Biological safety cabinet (Nuaire Cell Gard Class II)

- Vacuum pump (Integra Vacusafe)

- Ice and ice bucket

- Sterile $1.5 \mathrm{ml}$ Eppendorf safelock tubes (Fisher Scientific Cat. No. 0030120086)

- Sterile $15 \mathrm{ml}$ falcon tubes (Corning 430791)

\section{REAGENT SETUP}

CRITICAL: All tissue culture reagents must be kept sterile and prepared in a Biological safety cabinet suitable for tissue culture (see EQUIPMENT).

FBS 
Heat-inactivate the FBS by incubation for 30 minutes in a water bath at $56^{\circ} \mathrm{C}$. Filter-aliquot the inactivated serum using sterile syringe filters $(0.2 \mu \mathrm{m}, 33 \mathrm{~mm})$ and store in $40 \mathrm{ml}$ volumes at $-20^{\circ} \mathrm{C}$ for up to six months.

\section{Apo-transferrin solution}

To prepare a stock apo-transferrin solution, dissolve $100 \mathrm{mg}$ of powder in $10 \mathrm{ml}$ of sterile autoclaved water. Spin the solution at $1100 \mathrm{~g}$ for 3 minutes at room temperature, then transfer to a fresh sterile $1.5 \mathrm{ml}$ Eppendorf tube. Filter-aliquot into $1 \mathrm{ml}$ volumes and store at $-20^{\circ} \mathrm{C}$ for up to eight weeks.

\section{Progesterone}

To prepare a stock progesterone solution, dissolve $0.006 \mathrm{~g}$ of powder in $10 \mathrm{ml}$ of Ethanol. Filter-aliquot this solution into sterile $1.5 \mathrm{ml}$ Eppendorf tubes and cover lids of tubes with parafilm to prevent evaporation. Store at $-20^{\circ} \mathrm{C}$ for up to six months.

\section{Sodium selenite}

To prepare a stock sodium selenite solution, dissolve $0.006 \mathrm{~g}$ of powder in $10 \mathrm{ml}$ of sterile autoclaved water. Filter-aliquot this solution into sterile $1.5 \mathrm{ml}$ Eppendorf tubes and store at $-20^{\circ} \mathrm{C}$ for up to six months.

\section{Putrescine dihydrochloride}

To prepare a stock putrescine dihydrochloride solution, dissolve $0.8 \mathrm{~g}$ of powder in $5 \mathrm{ml}$ of sterile autoclaved water. Filter-aliquot this solution into sterile $1.5 \mathrm{ml}$ Eppendorf tubes and store at $-20^{\circ} \mathrm{C}$ for up to six months.

\section{FGF-4 solution reconstitution}

Reconstitute the lipophilized FGF-4 according to the manufacturer's instructions. Prepare a 1000x $(25 \mathrm{ug} / \mathrm{ml})$ stock solution by dilution using PBS 0.1\% BSA (wt/vol). Aliquot into sterile $1.5 \mathrm{ml}$ Eppendorf tubes and store at $-80^{\circ} \mathrm{C}$ for up to eight weeks. Do not re-freeze after thawing.

\section{Heparin solution reconstitution}

Prepare a $1000 \times(1 \mathrm{mg} / \mathrm{ml})$ stock solution (wt/vol) of heparin solution by dissolving $10 \mathrm{mg}$ heparin in $10 \mathrm{ml}$ of PBS. Aliquot into sterile $1.5 \mathrm{ml}$ Eppendorf tubes and store at $-80^{\circ} \mathrm{C}$ for up to six months. Do not re-freeze after thawing.

\section{Gelatin solution}

To prepare a $0.1 \%$ gelatin solution $(\mathrm{wt} / \mathrm{vol}$ ) dissolve $1 \mathrm{~g}$ of gelatin in $100 \mathrm{ml}$ of water and autoclave the solution. Store at $4^{\circ} \mathrm{C}$ for 1 month.

\section{Feeder Cell 'FC' Medium}

Prepare a solution comprising the following: DMEM supplemented with 15\% FBS (vol/vol), 2mM Lglutamine, $0.1 \mathrm{mM} 2-\mathrm{ME}, 0.1 \mathrm{mM}$ NEAA, $1 \mathrm{mM}$ sodium pyruvate, and $1 \%$ penicillin-streptomycin (vol/vol). Store at $4^{\circ} \mathrm{C}$ and use within 1 month. 


\section{FC 2iLIF Medium}

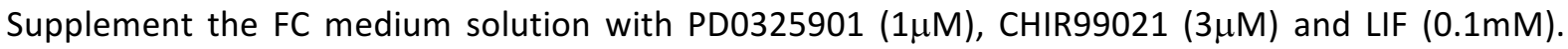
Store at $4^{\circ} \mathrm{C}$ and use within 1 week.

\section{N2 supplement}

Prepare a solution comprising the following: DMEM/F-12 supplemented with $10 \%$ human apotransferrin solution (vol/vol), 10\% Bovine Albumin Fraction $\mathrm{V}$ ( $\mathrm{vol} / \mathrm{vol}), 25 \%$ human insulin solution, putrescine dihydrochloride $(1611 \mu \mathrm{g} / \mathrm{ml})$, sodium selenite $(0.6 \mu \mathrm{g} / \mathrm{ml})$ and progesterone $(0.6 \mu \mathrm{g} / \mathrm{ml})$. Filter-aliquot this solution into sterile $0.5 \mathrm{ml}$ Eppendorf tubes and store at $-20^{\circ} \mathrm{C}$ for up to 1 month. Avoid repeated freeze-thaw cycles.

\section{N2B27}

Prepare a solution comprising the following: 50\% DMEM/F-12 (vol/vol) and 50\% Neurobasal A (vol/vol), supplemented with $2 \mathrm{mM}$ L-glutamine, 0.1mM 2ME, 0.5x N2 supplement, 1x B27 supplement. Store at $4^{\circ} \mathrm{C}$ and use within 2 weeks.

\section{N2B27 2iLIF}

Supplement the N2B27 solution with PD0325901 (1 $\mu \mathrm{M})$, CHIR99021 $(3 \mu \mathrm{M})$ and LIF (0.1mM). Store at $4^{\circ} \mathrm{C}$ and use within 1 week.

\section{ETS-embryo medium}

Prepare a solution comprising the following: 40\% 'MegaCell' RPMI (vol/vol), 25\% DMEM/F-12 (vol/vol) and $25 \%$ Neurobasal A (vol/vol) supplemented with 10\% FBS (vol/vol), $2 \mathrm{mM}$ L-glutamine, 0.1mM 2ME, $0.5 \mathrm{mM}$ sodium pyruvate, $0.25 \times \mathrm{N} 2$ supplement (made in house), $0.5 \times \mathrm{B} 27$ supplement, plus $12.5 \mathrm{ng} / \mathrm{ml}$ FGF4 and 500ng/ml heparin. Also add $1 \%$ penicillin-streptomycin (vol/vol). Store at $4^{\circ} \mathrm{C}$ and use within 1 week. Alternatively, the complete formulation can be purchased as a ready-to-use formula from Cell Guidance Systems, (Cat. No: M13-25)

\section{TS cell medium}

Prepare a solution comprising the following: 'MegaCell' RPMI with 20\% FBS (vol/vol), $2 \mathrm{mM} \mathrm{L-}$ glutamine, $0.1 \mathrm{mM} 2-\mathrm{ME}, 1 \mathrm{mM}$ sodium pyruvate, and $1 \%$ penicillin-streptomycin (vol/vol). Store at $4^{\circ} \mathrm{C}$ and use within 1 month.

\section{TSF4H medium}

Supplement the TS cell medium solution with $25 \mathrm{ng} / \mathrm{ml}$ of recombinant FGF4 and $500 \mathrm{ng} / \mathrm{ml}$ of heparin. Store at $4^{\circ} \mathrm{C}$ and use within 1 week.

\section{4\% PFA solution}

Dilute the $16 \%$ PFA solution four times in PBS to produce $4 \%$ PFA (vol/vol). Store at $4^{\circ} \mathrm{C}$ and use within 1 week.

\section{Blocking buffer for antibody incubations}


Prepare a solution of PBS with $3 \% \mathrm{BSA}$ (wt/vol). Store at $4^{\circ} \mathrm{C}$ and use within 1 week.

\section{Box 1: Preparation of mitotically inactivated mouse embryonic fibroblasts (MEFs)}

A brief procedure follows, more detailed instructions can be be found in Nagy et al. ${ }^{35}$.

Additional materials required:

CBA/C57BI6 (F1) mice

CAUTION: Experiments using mice must conform to Institutional and National regulations. We obtained approval to use the procedure below from Animals (Scientific Procedures) Act 1986 Amendment Regulations 2012 following ethical review by the University of Cambridge Animal Welfare and Ethical Review Body (AWERB).

Mitomycin-C (Sigma-Aldrich Cat. No. M4287)

DNasel (New England Biolabs Cat. No. M0303S)

Procedure:

i) Mate $\mathrm{CBA} / \mathrm{C} 57 \mathrm{BI}$ (F1) mice and sacrifice pregnant females after 13 or 14 days post coitum.

CRITICAL: Carry out all dissection steps in a tissue culture hood under aseptic conditions using sterile instruments.

ii) Dissect out uterine horns in sterile PBS, and remove embryos using fine forceps.

iii) Dissect away the brain and red organs from each embryo, then mince the tissue using a razor blade until it can be pipetted.

iv) Digest the minced tissue using $0.05 \%$ trypsin-EDTA and 100ul of DNasel per embryo, and incubate for 15 minutes at $37^{\circ} \mathrm{C}$.

v) Inactivate the trypsin using freshly prepared FC medium, and transfer the cell-suspension to a falcon tube.

vi) Centrifuge at $300 \mathrm{~g}$ for $5 \mathrm{~min}$, then subsequently remove the supernatant and resuspend the cells in fresh FC medium.

vii) Plate the cells on gelatine coated tissue culture dishes and culture overnight at 5\% $\mathrm{CO} 2$ and $37^{\circ} \mathrm{C}$.

viii) Change the medium the following day to remove dead and unattached cells.

ix) Expand the cells until confluent, and then inactivate using Mitomycin-C at 10 $\mathrm{gg} / \mathrm{ml}$ suspended in PBS. 
PAUSEPOINT: Once inactivated, MEFs can be kept in the incubator for up to 2 weeks until needed, if medium is changed regularly.

END BOX 1

\section{Box 2: Derivation of Trophoblast stem cells (TSCs)}

Trophoblast stem cells can be derived from the mouse embryo at blastocyst or early postimplantation stages. Steps for derivation from pre-implantation blastocysts are outlined below, but more detailed protocols are also available ${ }^{36}$.

\section{Additional materials required}

Mice of the desired strain. We have used CBA $\times$ C57BI/6 (F1 mice) and transgenic mice of mixed genetic background. Please note that ESCs and TSCs do not need to be derived from the same mouse strain to form ETS-embryos. Experiments using mice must conform to Institutional and National regulations. We obtained approval for these experiments from The Animals (Scientific Procedures) Act 1986 Amendment Regulations 2012 following ethical review by the University of Cambridge Animal Welfare and Ethical Review Body (AWERB).

M2 medium (Sigma, M7167)

Procedure:

i) Before starting, prepare 'conditioned medium' by culturing MEFs in TS medium for 3 days. Collect the medium and store for use during derivation.

ii) Plate MEFs on 4-well tissue culture plates at a density of $4 \times 10^{4}$ cells per well and culture in TS cell medium overnight at $5 \% \mathrm{CO} 2$ and $37^{\circ} \mathrm{C}$.

iii) Mate mice and sacrifice pregnant females after 4.5 days post coitum to obtain preimplantation blastocysts.

iv) Dissect out the uterus, flush uterine horns using M2 medium, and collect the blastocyst stage embryos.

v) Replace the TS cell medium on the MEFs with TSF4H medium and seed one blastocyst per well in the four-well plates covered with MEFs.

vi) Culture overnight at $5 \% \mathrm{CO} 2$ and $37^{\circ} \mathrm{C}$, and allow blastocysts to attach to the MEFs and form outgrowths.

vii) Replace the medium each day. 
viii) Once the outgrowths have expanded, aspirate the medium and wash with PBS, then dissociate the outgrowths using 0.05\% trypsin-EDTA. Incubate for 10 minutes at 5\% CO2 and $37^{\circ} \mathrm{C}$.

ix) Inactivate the trypsin using $70 \%$ conditioned medium, plus $1.5 \mathrm{x}$ TSF4H (TS medium containing $37.5 \mathrm{ng} / \mathrm{ml} \mathrm{FGF4} \mathrm{and} 1.5 \mathrm{\mu g} / \mathrm{ml}$ heparin) medium then disaggregate the outgrowths by pipetting up and down.

x) Incubate at $5 \% \mathrm{CO} 2$ and $37^{\circ} \mathrm{C}$, and change the medium every other day until TSC colonies begin to appear. The number of days for cells to appear will vary (usually from 6-10 days), but epithelial colonies with clear boundaries should form. Cells other than TSCs may also appear, in which case, pick the colonies of TSCs using a pipette and re-plate in new four well-plates coated with MEFs and culture at $5 \% \mathrm{CO} 2$ and $37^{\circ} \mathrm{C}$ in $\mathrm{TSF} 4 \mathrm{H}$ medium.

xi) Continue to expand the TSCs. On average, cells need to be passaged every 3-4 days, or when wells are $80 \%$ confluent. Cultures should be split at a ratio of 1:10. To passage TSCs: incubate with $0.05 \%$ trypsin- EDTA for 3-5 minutes; neutralise with an equal volume of TSC medium; gently disaggregate cells with a pipette; spin down cells (1000g for 3 minutes); resuspend in TSC medium; and plate on a fresh plate of mitotically inactivated MEFs. ${ }^{16}$

xii) TSCs can be cultured longer term on MEFs or can be cultured in MEF-free conditions on gelatin-coated tissue culture plates by keeping the cells in MEF-conditioned TS cell medium (more details can be found in Tanaka, $2006^{36}$ or Rossant, $2006^{37}$ ) As a third alternative, TS cells can also be maintained in completely defined conditions detailed protocols to maintain TSCs in this way have been developed by Kubaczka et al, $2014^{38}$ and by Ohinata et al, $2014^{39}$. TSCs maintained in all conditions are suitable for generating ETSembryos.

PAUSEPOINT TSCs can be frozen for storage by freezing at $-80^{\circ} \mathrm{C}$ in TS freezing medium (10\% DMSO, 70\% TS cell medium, 20\% FBS)

\section{END BOX 2}

\section{Box 3: Derivation of ESCs}

This is briefly described below. More detailed protocols for how to derive ESCs from mouse embryos are available should further guidance be required ${ }^{34}$.

Additional materials required: 
Female Mice of the desired strain. To date, we have used 129 ESCs, E14 ESCs, and have derived ESCs from transgenic mice of a mixed genetic background. All these strains are suitable to generate ETSembryos. Experiments using mice must conform to Institutional and National regulations. We obtained approval for these experiments from the Animals (Scientific Procedures) Act 1986 Amendment Regulations 2012 following ethical review by the University of Cambridge Animal Welfare and Ethical Review Body (AWERB).

Procedure:

i) Recover blastocysts from pregnant female mice and seed on inactivated MEF feeder cells in FC2iLIF medium.

ii) Culture overnight at $5 \% \mathrm{CO} 2$ and $37^{\circ} \mathrm{C}$.

iii) Allow the blastocysts to hatch, attach to the MEFs and form outgrowths. This usually takes 48 hours.

iv) Once the outgrowths have expanded, dissociate these by washing with PBS and incubating with $0.05 \%$ Trypsin-EDTA for 10 minutes.

v) Inactivate the trypsin with fresh FC2iLIF medium and disaggregate the outgrowths by pipetting up and down.

vi) Culture at $5 \% \mathrm{CO} 2$ and $37^{\circ} \mathrm{C}$, changing the medium every other day. ESC colonies should appear within 1 week of culture.

PAUSEPOINT: ESCs can be frozen for storage by freezing at $-80^{\circ} \mathrm{C}$ in $\mathrm{FC}$ freezing medium (10\% DMSO, 70\% FC medium, 20\% FBS)

END BOX 3

\section{PROCEDURE}

\section{Cell culture TIMING- minimum of 1 week}

CAUTION: The cell lines used in your research should be regularly checked to ensure they are authentic and are not infected with mycoplasma.

1) Establish both TSC and ESC cultures by carrying out options A and B. Manage ESC and TSC culture and passaging so that passaging-days become synchronised. MEF Feeder cells need not be removed to begin the ETS-embryo experiment. Feeder-free conditions are preferable for ESC culture, but MEFs will not contaminate the culture if they are still present when you start the experiment. When ESCs and TSCs are confluent (80\%) on the same day, proceed to the next step to generate ETS embryos.

A) TSC culture 
i. Plate mitotically inactivated mouse embryonic fibroblasts (MEFs) in a six-well tissue culture plate at half the density normally used for ESC culture $\left(1 \times 10^{5}\right.$ cells $\left./ \mathrm{ml}\right)$. Culture in FC medium at $5 \% \mathrm{CO} 2$ and $37^{\circ} \mathrm{C}$ for at least 24 hours, to allow the MEFs to attach to the plate.

ii. Thaw a vial of frozen TSCs as quickly as possible and re-suspend in TSF4H medium for culture. Remove the FC medium from the MEF plate and seed the TSC suspension on top of the MEFs (Typically, $\sim 1-3 \times 10^{5}$ TSCs can be plated per well of a six-well plate).

iii. Culture overnight at $5 \% \mathrm{CO} 2$ and $37^{\circ} \mathrm{C}$ to allow the TSCs to attach, then replace the medium with fresh TSF4H medium.

\section{? TROUBLESHOOTING}

iv. Continue to culture the cells, changing the culture medium every two days. Passage the cells as described in Box 2. CRITICAL STEP TSCS can be passaged up to 20 times for use in this protocol. Cells which have been passed a large number of times may have developed chromosomal abnormalities and should not be used in the culture.

v. Proceed to step 2 when you have plates of TSC and ESC cells (from option B) that are both $80 \%$ confluent.

\section{B) ESC culture}

i. Cover the wells of a 12 -well tissue culture plate with $0.01 \%$ gelatin $(\mathrm{wt} / \mathrm{vol}$ ) solution overnight.

ii. Thaw a vial of frozen ESCs as quickly as possible and re-suspend in the medium in which the cells were derived (FC2iLIF or N2B27 2iLIF).

\section{? TROUBLESHOOTING}

iii. Remove the excess gelatin from one well of the 12-well plate and seed the ESCs. Typically, $\sim 1.5 \times 10^{5}$ ESCs can be plated per well of the 12-well plate. Incubate the ESCs overnight to attach, then replace the medium with fresh FC2iLIF or N2B272iLIF.

iv. Culture cells at $5 \% \mathrm{CO} 2$ and $37^{\circ} \mathrm{C}$, changing the medium every other day, and inspecting the cultures each day to ensure cells are heathy. Healthy ESCs should form domed, bright colonies with clear boundaries. Passage the ESCs every two days, or when the well reaches confluency. To passage cells: incubate with $0.05 \%$ trypsin-EDTA for a maximum of 5 minutes; neutralise with an equal volume of FC medium ; gently disaggregate cells with a pipette; spin down cells (1000g for 5 minutes); resuspend in FC2iLIF or N2B272iLIF; and plate on a fresh gelatin coated plate $^{28}$ 
CRITICAL STEP ESCS can be passaged up to 20 times for use in this protocol. Cells which have been passed a large number of times may have developed chromosomal abnormalities and should not be used in the culture.

vi. Proceed to step 2 when you have plates of ESC and TSC cells (from option A) that are both $80 \%$ confluent.

\section{D embedding of ESCs and TSCs TIMING 1 hour}

CRITICAL To minimise time out-of-the-incubator, it is recommended to process ESCs and TSCs in parallel (steps 2-7). This can be done by a single researcher however if this is not possible, then it is recommended to trypsinize and re-suspend the ESCs first, then keep them in PBS under the tissue culture hood whilst working with the TSCs.

CRITICAL If it is useful to have all the cells in the same plane (for example when imaging multiple structures) a '3D on top' approach can be used, as described in Box 1.

2) Wash ESCs from step 1 with Phosphate Buffered Saline (PBS) and treat with 0.05\% trypsinEDTA for $3-4$ minutes at $37^{\circ} \mathrm{C}$.

3) Inactivate the trypsin using FC medium to obtain a single-cell suspension, and collect in a $15 \mathrm{ml}$ falcon tube.

CRITICAL STEP: ESCs should be dissociated to single cells by pipetting. The ESCs can be left in suspension at room temperature under the tissue culture hood whilst working with the TSCs.

4) Wash TSCs from step 1 with PBS and then treat with $0.05 \%$ trypsin-EDTA for 4 minutes at $37^{\circ} \mathrm{C}$.

5) Inactivate the trypsin using TS medium to obtain small clumps of TSCs in suspension. Collect the suspension in a $15 \mathrm{ml}$ falcon tube.

CRITICAL STEP: TSCs should be pipetted gently 2-3 times using a wide pipette tip just as a P1000 to preserve small clumps.

\section{? TROUBLESHOOTING}

6) If two centrifuges are available, one centrifuge can be used to work with ESCs while another can be used for TSCs in parallel. Centrifuge ESCs for 5 minutes at $1000 \mathrm{~g}$ at room temperature. and centrifuge the TSC suspension for $3-4$ minutes at $1000 \mathrm{~g}$ at room temperature.

7) After centrifugation of ESCs, resuspend the cells in PBS and centrifuge a second time to 'wash' the PD0325901, CHIR99021 and LIF from the ESCs.

CRITICAL STEP: Washing of ESCS is important to remove 2iLIF.

8) After centrifugation of TSCs, remove the supernatant, and resuspend the pellet gently in RPMI/FBS media.

CRITICAL STEP The handling of TSCs should be gentle to preserve small clumps of 2 to 8 cells. The size and number of cells in TSC clumps can be checked under the tissue-culture 
microscope at each stage of the procedure. Over-washing can reduce viability of TSCs, so avoid this.

9) After the second centrifugation of ESCs is complete, resuspend once again in PBS and centrifuge a third time. TSCs can be left at room temperature in the tissue culture hood while ESCs are centrifuged for the third time.

10) After the third centrifugation of ESC, remove the supernatant and resuspend the cell pellet in PBS to obtain a single cell suspension.

11) Using a haemocytometer, count $\sim 3-5,000$ ESCs and 3-5,000 TSC clumps and mix in a $1: 1$ suspension in a fresh $15 \mathrm{ml}$ falcon tube.

12) Centrifuge the mix for 3-4 minutes at $1000 \mathrm{~g}$ at room temperature, then remove the supernatant carefully using a P200 pipette, without disturbing the cell pellet.

\section{D embedding of ESC and TSC TIMING}

13) Resuspend the pellet in liquid Matrigel ( $\sim 100$ ul for all 8 wells of the $\mu$-plate (Ibidi)).

CRITICAL STEP: The use of growth-factor reduced Matrigel is recommended. This should be batch tested on cells before generating ETS embryos.

CRITICAL STEP: Thaw Matrigel on ice. Matrigel must be kept on ice in order to prevent solidification during the cell preparation.

CRITICAL STEP: Pipette tips for resuspending the cells should also be kept at $4{ }^{\circ} \mathrm{C}$ in order to prevent solidification of Matrigel.

14) Plate the Matrigel-cell suspension in small (10-20 $\mu$ l) drops on $\mu$-plates (Ibidi) and allow the matrix to solidify for 2 minutes at $37^{\circ} \mathrm{C}$. Plate one drop per well of the plate.

Once the Matrigel is solidified, fill each culture well with ETS-embryo medium and culture in $5 \% \mathrm{CO} 2$ at $37^{\circ} \mathrm{C}$. ? TROUBLESHOOTING

\section{Co-culture of ESCs and TSCs to generate ETS embryos TIMING- maximum 6 days}

15) Culture cells in ETS-embryo medium for up to six days after plating. Inspect cultures every day to monitor ETS-embryo formation. Change the medium every $48 \mathrm{~h}$ or when cells deplete nutrients, as indicated by the phenol red present in the medium. When medium requires changing, the medium will change colour from a red-pink colour to a 'straw-like' colour. Proceed to next step at appropriate time point for your experiment (4, 5 or 6 of culture).

\section{? TROUBLESHOOTING}

\section{Immunofluorescence analysis of ETS embryos TIMING- 2-3 days}

16) Remove the medium from ETS-embryo cultures and rinse with PBS, then fix for 15 minutes at room temperature in $4 \%$ ice-cold paraformaldehyde (PFA) in PBS (vol/vol). The PFA will lead 
to the collapse of the Matrigel droplets, and structures will stick to the optical plastic/ glass of the culture dish. ? TROUBLESHOOTING

17) After fixation, rinse samples twice in PBS for 5 minutes each, then permeabilise at room temperature for 10 minutes in $0.3 \%$ Triton-X-100, 0.1\% glycin in PBS (vol/vol).

PAUSE POINT: Following fixation samples can be processed immediately for immunofluorescence staining or left in PBS up to 2 weeks at $4^{\circ} \mathrm{C}$.

18) Rinse again in PBS, then perform primary antibody incubation at $4^{\circ} \mathrm{C}$ overnight in blocking buffer. Examples of primary antibodies we have used successfully and recommended dilutions are given in the materials.

19) The following day, remove the primary antibody in blocking buffer and wash samples three times for 5 minutes each in PBS at room temperature to remove unbound antibody.

20) Perform secondary antibody incubation with an appropriate antibody in blocking buffer at room temperature for 2 hours.

PAUSE POINT: Incubation with secondary antibody can also be performed at $4^{\circ} \mathrm{C}$ overnight.

21) After incubation, wash the samples three times in PBS for 5 minutes each at room temperature, then incubate in DAPI plus PBS for a further 1 hour at room temperature.

22) Remove the DAPI/PBS solution, add PBS into plates and image ETS-embryo structures in situ in the optical plates using a confocal microscope (Leica or equivalent). ? TROUBLESHOOTING

\section{BOX 4: ‘3D on top' protocol for ETS-embryo generation}

For imaging a high number of structures, it is useful to have all the cells in the same plane .For this purpose, a '3D on top' approach (modified from Lee et al., ${ }^{40}$ ) can be used. This method is amenable to the use of different optical plates of different sizes, depending on the purpose of the experiment. The modifications to the main procedure required to establish the '3D on top' approach for 8-well $\mu$ plates (Ibidi) is described below and coating, seeding and media volumes for plates of different sizes is given in Supplementary Table 1.

1) Thaw Matrigel on ice before beginning the experiment.

2) Cover all 8-wells of $\mu$-plates (Ibidi) evenly with $35 \mathrm{ul}$ of liquid Matrigel to cover the surface.

3) Place the plate in the incubator at $37^{\circ} \mathrm{C}$ for two minutes, or until the Matrigel has solidified. 
4) Add 100ul of PBS to each well of the Matrigel-coated plate and place in the incubator until ready to use. This step prevents the Matrigel from drying out.

5) Trypsinize and wash cells as described in steps 2-11 of the main procedure to yield a 1:1 mixture of ESCs and TSC clumps in PBS.

6) Remove the PBS from each well of the plate and seed the 1:1 mix of ESCs and TSCs in a 25ul volume and allow them to attach for 10-15 minutes in the incubator.

CRITICAL STEP: The cells must be allowed to attach in the incubator before proceeding. If cells do not attach, they will be removed during later steps and ETS embryos will not be generated.

7) Prepare a mixture of ETS medium and $10 \%$ liquid Matrigel ( $\mathrm{vol} / \mathrm{vol}$ ), and keep this on ice until ready to use.

8) Once the cells have attached, remove the extra PBS with a vacuum pump or carefully using a pipette and fill each well of the plate with a mixture of ETS medium and 10\% (vol/vol) Matrigel.

9) Culture cells in $5 \% \mathrm{CO} 2$ at $37^{\circ} \mathrm{C}$ for up to six days after plating. Inspect cultures every day to monitor ETS-embryo formation.

END BOX

\section{? TROUBLESHOOTING}

See Table 1 for troubleshooting guidance.

Table 1: Troubleshooting

\begin{tabular}{|c|c|c|c|}
\hline Step & Problem & $\begin{array}{l}\text { Possible } \\
\text { Reason }\end{array}$ & Possible solution \\
\hline $\begin{array}{l}\text { Step } \\
\text { 1Aiii, } \\
\text { 1Bii }\end{array}$ & $\begin{array}{l}\text { Low rate } \\
\text { and density } \\
\text { of growth of } \\
\text { structure in } \\
\text { ECM }\end{array}$ & $\begin{array}{l}\text { Low rate of cell } \\
\text { survival/ } \\
\text { recovery after } \\
\text { thawing }\end{array}$ & $\begin{array}{l}\text { Keep cells in adherent culture for at least one passage after } \\
\text { thawing to increase the number of cells before attempting an } \\
\text { ETS-embryo experiment }\end{array}$ \\
\hline $\begin{array}{l}\text { Step } \\
\text { 1Aiii, } \\
1 \text { Bii }\end{array}$ & $\begin{array}{l}\text { Formation } \\
\text { of } \\
\text { disorganised } \\
\text { structures } \\
\text { which do }\end{array}$ & $\begin{array}{l}\text { Use of } \\
\text { differentiated } \\
\text { ESCs and TSCS } \\
\text { in the protocol }\end{array}$ & $\begin{array}{l}\text { Maintain cells in conditions to preserve stem cell character } \\
\text { and prevent differentiation. Inspect plates for morphological } \\
\text { evidence of differentiation (altered colony morphology in ESC } \\
\text { cultures and presence of trophoblast giant cells in TSC }\end{array}$ \\
\hline
\end{tabular}




\begin{tabular}{|c|c|c|c|}
\hline & $\begin{array}{l}\text { not } \\
\text { resemble } \\
\text { the egg } \\
\text { cylinder }\end{array}$ & & $\begin{array}{l}\text { cultures) and do not use cells in the protocol that are } \\
\text { differentiated. }\end{array}$ \\
\hline $\begin{array}{c}\text { Step } \\
5\end{array}$ & $\begin{array}{l}\text { Low rate of } \\
\text { TSC survival } \\
\text { in ECM }\end{array}$ & $\begin{array}{l}\text { TSC viability } \\
\text { can be } \\
\text { compromised } \\
\text { if dissociated } \\
\text { to single cells }\end{array}$ & $\begin{array}{l}\text { Preserve small clumps by dissociating pellets gently after } \\
\text { centrifugation. If TSCs are prone to dissociating into single } \\
\text { cells, suspend cells using a wide P1000 filter tip to avoid } \\
\text { complete dissociation of clumps. }\end{array}$ \\
\hline $\begin{array}{c}\text { Step } \\
14\end{array}$ & $\begin{array}{l}\text { Low rate of } \\
\text { ETS embryo } \\
\text { generation } \\
\text { and survival }\end{array}$ & $\begin{array}{l}\text { If ESCs and } \\
\text { TSCs remain } \\
\text { out of culture } \\
\text { for prolonged } \\
\text { time than } \\
\text { recommended, } \\
\text { ETS-embryo } \\
\text { generation can } \\
\text { be } \\
\text { compromised } \\
\text { due to the } \\
\text { decreased } \\
\text { survival of the } \\
\text { cells. }\end{array}$ & $\begin{array}{l}\text { It is recommended to work with ESCs and TSCs in parallel to } \\
\text { generate the mixed cell suspension in order to minimise the } \\
\text { time that cells are handled in suspension. If this is not possible } \\
\text { keeping the cells in buffered-based media can be used. }\end{array}$ \\
\hline $\begin{array}{c}\text { Step } \\
15\end{array}$ & $\begin{array}{l}\text { High rate of } \\
\text { cell death } \\
\text { among ESCs } \\
\text { in the first } \\
48 \mathrm{~h} \text { during } \\
\text { ETS embryo } \\
\text { generation } \\
\text { and culture }\end{array}$ & $\begin{array}{c}\text { Medium } \\
\text { change is too } \\
\text { infrequent }\end{array}$ & $\begin{array}{l}\text { Change the medium every day if high levels of cell death is } \\
\text { observed and the media pH (indicated by phenol red) changes } \\
\text { rapidly. }\end{array}$ \\
\hline
\end{tabular}




\begin{tabular}{|c|c|c|c|}
\hline $\begin{array}{c}\text { Step } \\
16\end{array}$ & $\begin{array}{c}\text { Matrigel } \\
\text { drops do } \\
\text { not collapse } \\
\text { upon } \\
\text { fixation }\end{array}$ & $\begin{array}{l}\text { PFA is too } \\
\text { dilute and not } \\
\text { kept ice-cold. } \\
\text { Or very few } \\
\text { cells have } \\
\text { survived in the } \\
\text { Matrigel. }\end{array}$ & $\begin{array}{c}\text { Ensure the PFA is kept on ice throughout the procedure and } \\
\text { that } 4 \% \text { PFA in PBS is used. }\end{array}$ \\
\hline $\begin{array}{c}\text { Step } \\
22\end{array}$ & $\begin{array}{c}\text { High } \\
\text { background } \\
\text { and non- } \\
\text { specific } \\
\text { fluorescence } \\
\text { is detected } \\
\text { upon } \\
\text { imaging }\end{array}$ & $\begin{array}{l}\text { Non-specific } \\
\text { antibody is } \\
\text { retained in the } \\
\text { samples due to } \\
\text { insufficient } \\
\text { washing }\end{array}$ & $\begin{array}{l}\text { Lengthen the time of washing steps to } 10 \mathrm{~min} \text { to ensure all } \\
\text { unbound primary and secondary antibody is removed. }\end{array}$ \\
\hline
\end{tabular}

TIMING

- Step 1 Cell culture $(A, B)$ Culture of ESCs and TSCs for experiment 1 week

- Steps 2-14 Embedding of ESCS and TSCs in ECM for co-culture 1 hour

- Step 15 Culture, monitoring and media changes 6 days

- Steps 16-22 Analysis of ETS-embryos by immunofluorescence 2-3 days

BOX 1 Steps 1-8 '3D on top' plating 1 hour

BOX 1 Step 9 Co-culture to generate ETS-embryos $~ 6$ days

\section{ANTICIPATED RESULTS}

ESCs and TSCs in close proximity should generate rosette-like structures that contact each other within $48 \mathrm{~h}$. After $72 \mathrm{~h}$ compound structures with a lumen in the ESC compartment should be present, and after 96h, the two tissue compartments should share a common cavity (Fig. 1). The efficiency of ETS-embryo formation is variable, with an average of $22 \%$ of structures per well forming embryo-like structures ${ }^{17}$ within 4 days of culture (400 structures were analysed in culture to calculate 
this value for efficiency, with 88 structures forming compound structures). At this stage, ETS-embryos vary slightly in the number of cells which make up the ESC and TSC compartments respectively, but are consistent in sharing a continuous cavity at the centre of the structure by $96 \mathrm{~h}$. Compound structures can be considered as representative ETS embryos therefore if they develop this continuous cavity and if ESC and if ESCS and TSC compartments occupy approximately $50 \%$ of total tissue volume each $^{17}$.

It is important that ESCs are cultured to preserve the naïve pluripotent state before use in the protocol. If ESCs are allowed to differentiate in culture, ETS-embryo generation can be compromised. Differentiated ESCs can give rise to ETS-embryos with disorganised ESC compartments, which fail to organise a columnar epithelium, or a continuous pro-amniotic cavity. Similar structure morphology can be seen when too much serum is used in the ETS-embryo medium. If differentiated TSCs are used in this protocol, compound structures fail to grow in culture.

If cell viability is good, $100 \%$ of ETS-embryos will express lineage markers determining the cell identity in discrete embryonic and extraembryonic compartments (Fig. 2). However, mesodermal specification among ETS-embryos is variable. We found that $66 \%$ of the structures in culture that can be considered as ETS-embryos will specify some cells as mesoderm (by the detection of T/Brachury expression) somewhere in the ESC compartment of the structure. $43 \%$ of ETS embryos will specify mesoderm which is 'regionalised' in that it is localised to one side of the long axis of the structure within $4-5$ days. $34 \%$ of ETS-embryos fail to specify any T/Brachyury positive cells in the ESC compartment.

\section{ACKNOWLEDGEMENTS}

Research fellowship (grant no. 098287/Z/12/Z) and for a European Research Council grant (code: 669198) awarded to M.Z.-G. to fund this work. We are also grateful for the BBSRC DTP studentship that supports S.E.H. and to the Scientific and Technological Research Council of Turkey, which supports B.S.'

\section{AUTHOR CONTRIBUTIONS}

S.E.H, B.S \& M.Z.G designed the protocol, carried out the work, analysed the results and prepared the manuscript. 


\section{COMPETING INTERESTS}

M.Z.-G. and S.E.H. declare that they are inventors on a patent application (1615343.9) submitted by Cell Guidance Systems (in which the University of Cambridge and the Wellcome Trust are beneficiaries) that covers the method and medium composition used to generate stem cell-derived embryos. B.S. declares no competing interests.

\section{REFERENCES}

1. Boroviak, T., Loos, R., Bertone, P., Smith, A. \& Nichols, J. The ability of inner-cell-mass cells to self-renew as embryonic stem cells is acquired following epiblast specification. Nat. Cell Biol. 16, 516-28 (2014).

2. Ying, Q.-L. \& Smith, A. G. Defined Conditions for Neural Commitment and Differentiation. Methods Enzymol. 365, 327-341 (2003).

3. Murry, C. E. \& Keller, G. Differentiation of Embryonic Stem Cells to Clinically Relevant Populations: Lessons from Embryonic Development. Cell 132, 661-680 (2008).

4. Irion, S., Nostro, M. C., Kattman, S. J. \& Keller, G. M. Directed differentiation of pluripotent stem cells: from developmental biology to therapeutic applications. Cold Spring Harb. Symp. Quant. Biol. 73, 101-110 (2008).

5. Hattori, N. Cerebral organoids model human brain development and microcephaly. Mov. Disord. 29, 185-185 (2014).

6. Eiraku, M. et al. Self-organizing optic-cup morphogenesis in three-dimensional culture. Nature 472, 51-6 (2011).

7. Xia, Y. et al. The generation of kidney organoids by differentiation of human pluripotent cells to ureteric bud progenitor-like cells. Nat. Protoc. 9, 2693-704 (2014).

8. Takasato, M. et al. Directing human embryonic stem cell differentiation towards a renal lineage generates a self-organizing kidney. Nat. Cell Biol. 16, 118-26 (2014).

9. Takasato, M., Er, P. X., Chiu, H. S. \& Little, M. H. Generation of kidney organoids from human pluripotent stem cells. Nat. Protoc. 11, 1681-1692 (2016).

10. Meinhardt, A. et al. 3D Reconstitution of the Patterned Neural Tube from Embryonic Stem Cells. Stem Cell Reports 3, 1-13 (2014).

11. ten Berge, D. et al. Wnt signaling mediates self-organization and axis formation in embryoid 
bodies. Cell Stem Cell 3, 508-18 (2008).

12. van den Brink, S. C. et al. Symmetry breaking, germ layer specification and axial organisation in aggregates of mouse embryonic stem cells. Development 141, 4231-42 (2014).

13. Warmflash, A., Sorre, B., Etoc, F., Siggia, E. D. \& Brivanlou, A. H. A method to recapitulate early embryonic spatial patterning in human embryonic stem cells. Nat. Methods 11, (2014).

14. Bedzhov, I. \& Zernicka-Goetz, M. Self-Organizing Properties of Mouse Pluripotent Cells Initiate Morphogenesis upon Implantation. Cell 156, 1032-44 (2014).

15. Arnold, S. J. \& Robertson, E. J. Making a commitment: cell lineage allocation and axis patterning in the early mouse embryo. Nat. Rev. Mol. Cell Biol. 10, 91-103 (2009).

16. Tanaka, S., Kunath, T., Hadjantonakis, A., Nagy, A. \& Rossant, J. Promotion of Trophoblast Stem Cell Proliferation by FGF4. Science (80-. ). 282, 2072-2075 (1998).

17. Harrison, S. E., Sozen, B., Christodoulou, N., Kyprianou, C. \& Zernicka-goetz, M. Assembly of embryonic and extra-embryonic stem cells to mimic embryogenesis in vitro. Science (80-. ). (2017). doi:10.1126/science.aal1810

18. Wilkinson, D. G. B. S. H. B. G. Expression of the mouse T gene and its role in mesoderm formation. Lett. To Nat. 346, 183-187 (1990).

19. Scialdone, A. et al. Resolving early mesoderm diversification through single-cell expression profiling. Nature 535, 289-293 (2016).

20. Peng, G. et al. Spatial Transcriptome for the Molecular Annotation of Lineage Fates and Cell Identity in Mid-gastrula Mouse Embryo. Dev. Cell 36, 681-697 (2016).

21. Winnier, G., Blessing, M., Labosky, P. A. \& Hogan, B. L. M. Bone morphogenetic protein-4 is required for mesoderm formation and patterning in the mouse. Genes Dev. 9, 2105-2116 (1995).

22. Lawson, K. A. et al. Bmp4 is required for the generation of primordial germ cells in the mouse embryo. Genes Dev. Dev. 13, 424-436 (1999).

23. Kreuter, J. Nanoparticles and microparticles for drug and vaccine delivery. J. Anat. $\mathbf{1 8 9}$ ( Pt 3, 503-505 (1996).

24. Kunath, T. et al. Imprinted X-inactivation in extra-embryonic endoderm cell lines from mouse blastocysts. Development 132, 1649-1661 (2005). 
25. Brown, K. et al. Extraembryonic endoderm (XEN) stem cells producefactors that activate heart formation. PLoS One 5, (2010).

26. Paca, A. et al. BMP signaling induces visceral endoderm differentiation of XEN cells and parietal endoderm. Dev. Biol. 361, 90-102 (2012).

27. Artus, J. et al. BMP4 signaling directs primitive endoderm-derived XEN cells to an extraembryonic visceral endoderm identity. Dev. Biol. 361, 245-262 (2012).

28. Ying, Q. et al. The ground state of embryonic stem cell self-renewal. Nature 453, 519-24 (2008).

29. Morris, S. a et al. Dynamics of anterior-posterior axis formation in the developing mouse embryo. Nat. Commun. 3, 673 (2012).

30. Bedzhov, I., Leung, C. Y., Bialecka, M. \& Zernicka-Goetz, M. In vitro culture of mouse blastocysts beyond the implantation stages. Nat. Protoc. 9, 2732-2739 (2014).

31. Fehling, H. J. et al. Tracking mesoderm induction and its specification to the hemangioblast during embryonic stem cell differentiation. Development 130, 4217-4227 (2003).

32. Payer, B. et al. Generation of stella-GFP transgenic mice: a novel tool to study germ cell development. Genesis 44, 75-83 (2006).

33. Rhee, J. M. et al. In vivo imaging and differential localization of lipid-modified GFP-variant fusions in embryonic stem cells and mice. Genesis 44, 202-218 (2006).

34. Behringer, R., Gertsenstein, M., Nagy, K. V. \& Nagy, A. Manipulating the Mouse Embryo: a Laboratory Manual 4th ed. (Cold Spring Harbor Laboratory Press, 2014).

35. Nagy, A., Gertsenstein, M., Vintersten, K. \& Behringer, R. Preparing Feeder Cell Layers from STO or Mouse Embryo Fibroblast (MEF) Cells: Treatment with Mitomycin C. CSH Protoc. 2006, (2006).

36. Tanaka, S. Derivation and culture of mouse trophoblast stem cells in vitro. Methods Mol. Biol. 329, 35-44 (2006).

37. Rossant, J. Culturing Trophoblast Stem (TS) Cell Lines. CSH Protoc. (2006). doi:10.1101/pdb.prot4406

38. Kubaczka, C. et al. Derivation and maintenance of murine trophoblast stem cells under defined conditions. Stem Cell Reports 2, 232-242 (2014). 
39. Ohinata, Y. \& Tsukiyama, T. Establishment of trophoblast stem cells under defined culture conditions in mice. PLoS One 9, (2014).

40. Lee, G. Y., Kenny, P. a, Lee, E. H. \& Bissell, M. J. Three-dimensional culture models of normal and malignant breast epithelial cells. Nat. Methods 4, 359-65 (2007).

\section{Figure legends}

Figure 1: Generation of polarised embryo-like structures from embryonic and extra-embryonic stem cells to form ETS-embryos. Schematic of the method used to generate ETS-embryos from ESCS and TSCs. (a) When $80 \%$ confluent, ESCS and TSCs are dissociated into single cells and small clumps respectively (Steps $2-10)$. Images from the haemocytometer square $(0.25 \times 0.25 \mathrm{~mm})$ to count single ESCs and TSC clumps (Step 11) Scale bar $=50 \mu \mathrm{m}$. (b) Mixing and resuspension of cells in liquid Matrigel (Steps 12,13) (c) Plate cell-Matrigel suspension in small drops, allow to solidify and culture in ETS medium (Step 14) (d)\&(e) The '3D on top' variation of culture (described in Box 4.) (f) Lowmagnification of confocal images of the culture plate at indicated time points showing the co-culture of CAG-GFP labelled ESCs and wild-type TSCs on Matrigel. Single ESCs and TSC clumps ( $3 \mathrm{~h}$, inset) grow to produce single spheroid of ESC or TSC colonies (24h, inset) which later come together (48-72h) and finally merge to generate embryo-like structures, ETS-embryos (96h). Scale bar $=50 \mu \mathrm{m}$. (g) Time scheme of ETS-embryo generation. (h) High-magnification of confocal snapshots of an ETS-embryo developing over $96 \mathrm{~h}$ from clumps of cells. Within $12 \mathrm{~h}$, the single ESC divides to give rise to a doublet and later a spheroid of polarised cells, which later merges with the TSCs to generate an ETS-embryo. The ESC compartment is labelled with a CAG-GFP membrane marker to demarcate it from the TSC compartment. Yellow dashed lines outline the cavity. Scale bar $=10 \mu \mathrm{m}$.

Figure 2: Molecular markers ascertaining to the lineage identity of the cell types in the embryonic and extra-embryonic compartments of polarised embryo-like structures, ETS-embryos. ETS-embryos cultured for 110h were analysed for ExE markers (EOMES, grey (a); TFAP2C, grey (b)); epiblast markers (SOX2, red (a); OTX2, red (b)) and mesoderm marker (T/Brachyury, green) by immunofluorescence. DAPI, blue; Scale bar $=20 \mu \mathrm{m}$.

Supplementary table 1: Table listing the approximate volumes of medium and Matrigel used with this method for different sizes and types of optical culture dish. 